\title{
Practical synchronization on complex dynamical networks via optimal pinning control
}

\author{
Kezan $\mathrm{Li}^{1}$, Weigang $\mathrm{Sun}^{2}$, Michael Small ${ }^{3}$, and Xinchu $\mathrm{Fu}^{4}$ \\ ${ }^{1}$ School of Mathematics and Computing Science, \\ Guangxi Key Laboratory of Cryptography and Information Security, \\ Guilin University of Electronic Technology, Guilin 541004, People's Republic of China \\ 2 Institute of Applied Mathematics and Engineering Computations, \\ Hangzhou Dianzi University, Hangzhou 310018, People's Republic of China \\ 3 School of Mathematics and Statistics, The University of Western Australia, Crawley, WA, 6009, Australia \\ ${ }^{4}$ Department of Mathematics, Shanghai University, Shanghai 200444, People's Republic of China
}

\begin{abstract}
We consider practical synchronization on complex dynamical networks under linear feedback control designed by optimal control theory. The control goal is to minimize global synchronization error and control strength over a given finite time interval, and synchronization error at terminal time. By utilizing the Pontryagin's minimum principle, and based on a general complex dynamical network, we obtain the optimal system to achieve the control goal. The result is verified by performing some numerical simulations on Star networks, Watts-Strogatz networks and BA networks. Moreover, by combining the optimal control and traditional pinning control, we propose an optimal pinning control strategy which depends on the network's topological structure. Obtained results show that the optimal pinning control is very effective for synchronization control in real applications.
\end{abstract}

PACS numbers: 05.45.Xt, 89.75.Hc

Since synchronization of complex networks has potential applications in various fields, it has been studied thoroughly and widely during the past few decades [1-4] (and many references therein). In order to realize various synchronization patterns, many synchronization control methods have been proposed, such as pinning control [5, 6], impulsive control [7], adaptive control [8], and so on. A general and common control goal is to realize complete synchronization, i.e., the synchronization error converges to zero as time $t \rightarrow+\infty$. Though these control methods can effectively achieve the synchronization goal, they generally need great control strength. In particular, these methods may be unfeasible when the control strength is limited to a small range. In order to achieve a balance between the control strength and synchronization goal, this paper will study network synchronization by applying a time-varying control via the optimal control theory to globally reduce the synchronization error and control strength. Noting that time interval for every complex dynamical network in real applications is usually finite, in this case, the control goal becomes minimizing the global synchronization error and control strength over the given finite time interval, and the synchronization error at terminal time. Actually, the expected synchronization becomes practical complete synchronization $[9,10]$ (or practical synchronization for short), where the synchronization error is limited globally to a small level.

Synchronization of master-slave systems via optimal control theory has been studied in recent years [11-13]. However, to the best of our knowledge, there are no reports of synchronization on complex dynamical networks via optimal control. So, based on a general dynamical complex network model, in this paper we will investigate its practical synchronization with optimal control. We find that the proposed optimal control method is more effective than linear feedback control with constant strength (i.e., constant control). To further improve the performance of optimal control, we introduce the optimal pinning control strategy, by which only a fraction of nodes in the network are controlled. These results are verified by numerical simulations.

The behaviour of the complex dynamical network under control is described by

$$
\left\{\begin{aligned}
\dot{x}_{i}(t)= & f\left(x_{i}(t)\right)+c \sum_{j=1}^{n} a_{i j} x_{j}(t) \\
& +u_{i}(t)\left(s(t)-x_{i}(t)\right) \\
\dot{s}(t)= & f(s(t))
\end{aligned}\right.
$$

where $x_{i}(t)=\left(x_{i 1}(t), x_{i 2}(t), \ldots, x_{i m}(t)\right)^{T} \in \mathbb{R}^{m}$ denote the state variables for $i=1,2, \ldots, n$. The initial condition of model (1) is set as

$$
\left\{\begin{array}{l}
x_{i}(0)=\theta_{i} \in \mathbb{R}^{m}, \quad i=1,2, \ldots, n, \\
s(0)=\theta_{n+1} \in \mathbb{R}^{m}
\end{array}\right.
$$

The nonlinear vector function $f(\cdot)$ defines the local dynamics of node $i$, and we assume that it is differentiable. The constant $c>0$ is the coupling strength. The coupling matrix $A=\left(a_{i j}\right) \in \mathbb{R}^{n \times n}$ with zero-sum rows shows the coupling configuration of the network. If nodes $i$ and $j$ are connected, then $a_{i j}=a_{j i}=1$; otherwise $a_{i j}=a_{j i}=0$. The diagonal elements of the coupling matrix $A$ are $a_{i i}=-\sum_{j=1, j \neq i}^{N} a_{i j}=-k_{i}, \quad i=1,2, \ldots, n$, where $k_{i}$ denotes the degree of node $i$. In this paper, we suppose that the matrix $A$ is irreducible. Let $u_{i}(t) \in \mathbb{R}^{+}$ denote the time-varying control strength. Note that if $u_{i}(t)$ are constant, synchronization of model (1) has been studied widely under linear feedback control method $[2,4]$. In this case, large control strengths are generally needed to drive all nodes toward the desired state $s(t)$. In order to decrease the control strength, timevarying adaptive control can be used [6, 14]. However, 
this method can not guarantee that the control strength reaches the optimal level to achieve the synchronization goal. So, how to realize its synchronization and decrease control strength at the same time from the view of optimization is still an open problem in complex networks. Under this general dynamical complex network (1), this paper will address this problem by utilizing the optimal control theory.

First, define synchronization error variables $e_{i}(t)=$ $x_{i}(t)-s(t)$ for $i=1,2, \ldots, n$, where $s(t)$ is the expected synchronization state for all nodes. Since both the control strength and time interval in real applications are usually finite, we define time-varying control strength $u_{i}(t) \in U_{a d}=\{\xi(t)$ is measurable, $0 \leq \xi(t) \leq$ $\left.b, t \in\left[0, t_{f}\right]\right\}$, where $b>0$ is upper bound of the control strength and $t_{f}>0$ is terminal time. Since our control goal is to decrease the global synchronization error and control strength, and the synchronization error at the terminal time, then a general objective function is set as

$J(u)=\frac{1}{2} \sum_{i=1}^{n}\left\{e_{i}^{T}\left(t_{f}\right) e_{i}\left(t_{f}\right)+\int_{0}^{t_{f}}\left[e_{i}^{T}(t) e_{i}(t)+\beta_{i} u_{i}^{2}(t)\right] d t\right\}$,

with Lagrangian $L=\frac{1}{2} \sum_{i=1}^{n}\left[e_{i}^{T}(t) e_{i}(t)+\beta_{i} u_{i}^{2}(t)\right]$, where parameters $\beta_{i}$ are all positive weights, $u(t)=$ $\left(u_{1}(t), u_{2}(t), \ldots, u_{n}(t)\right)$. In fact, the integral form in objective function (3) is generally chosen to study the synchronization on dynamical systems [11-13]. This kind of objective function can insure that the synchronization error is decreased in the whole time interval $\left[0, t_{f}\right]$ under the following optimal control. If we want to strengthen relatively the control of synchronization (i.e., weaken the consideration of control strength), then the weights $\beta_{i}$ should be decreased, and vice versa.

Therefore, the optimal control problem can be described as

$$
\mathrm{OCP}: \begin{cases}\min , & J(u(t)) \\ \text { s.t. } & u(t) \in U_{a d}^{n}\end{cases}
$$

The optimal solution $\left(x_{i}^{*}(t), u_{i}^{*}(t)\right)$ of OCP will be resolved by using the Pontryagins minimum principle [15]. Combining the standard optimality conditions and complex network structure, we develop a new formalization of network control in terms of optimal control. Define the Hamiltonian $H$ for OCP as

$$
H=L+\sum_{i=1}^{n} \lambda_{i}^{T}(t) \dot{x}_{i}(t)
$$

where $\lambda_{i}(t)=\left(\lambda_{i 1}(t), \lambda_{i 2}(t), \ldots, \lambda_{i m}(t)\right)^{T} \in \mathbb{R}^{m}$ are the adjoint variables determined by the following equations

$$
\begin{aligned}
\dot{\lambda}_{i}(t)= & -\left.\frac{\partial H}{\partial x_{i}(t)}\right|_{x_{i}(t)=x_{i}^{*}(t), u_{i}(t)=u_{i}^{*}(t)} \\
= & -e_{i}^{*}-\left[\frac{\partial f\left(x_{i}^{*}(t)\right)}{\partial x_{i}^{*}(t)}\right]^{T} \lambda_{i}(t)-c \sum_{j=1}^{n} a_{j i} \lambda_{j}(t) \\
& +u_{i}^{*}(t) \lambda_{i}(t)
\end{aligned}
$$
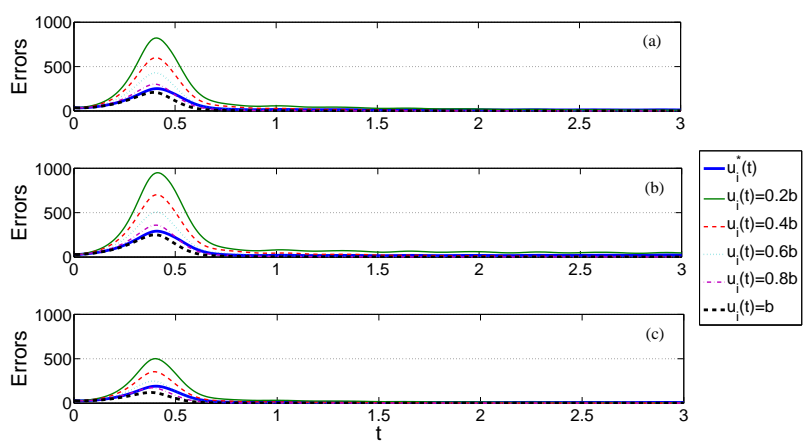

FIG. 1: (Color online) The synchronization errors $e(t)$ on different complex networks with different controls, where $e(t)=\sum_{i=1}^{n}\left\|e_{i}(t)\right\|_{2}$. With the parameters (i), (a),(b),(c) are obtained on Star network, Watts-Strogatz network, BA network, respectively.
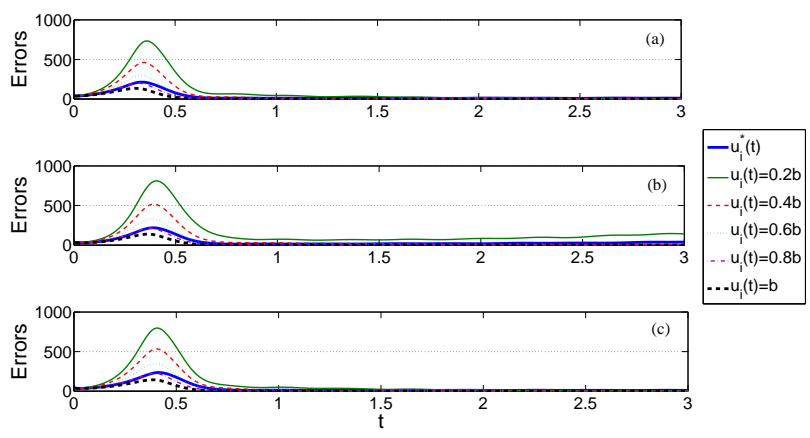

FIG. 2: (Color online) The synchronization errors $e(t)$ on different complex networks with different controls. With the parameters (ii), (a),(b),(c) are obtained on Star network, WattsStrogatz network, BA network, respectively.

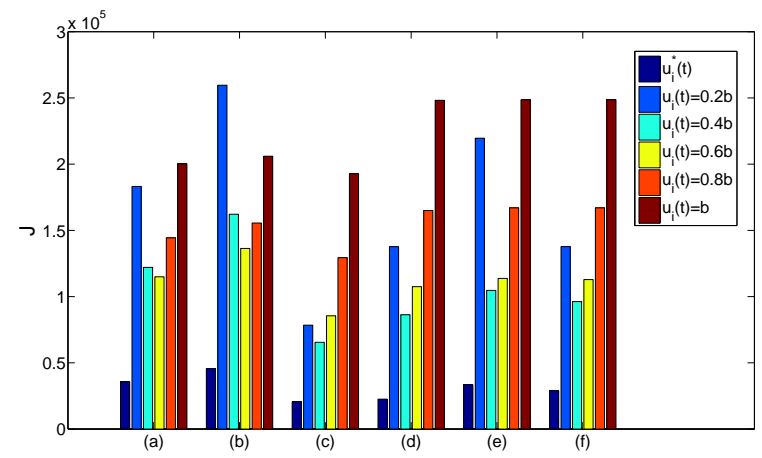

FIG. 3: (Color online) The values of the objective function (3) on different complex networks with different controls. With the parameters (i),(a),(b),(c) are obtained on Star network, Watts-Strogatz network, BA network, respectively; with the parameters (ii),(d),(e),(f) are obtained on Star network, Watts-Strogatz network, BA network, respectively. 

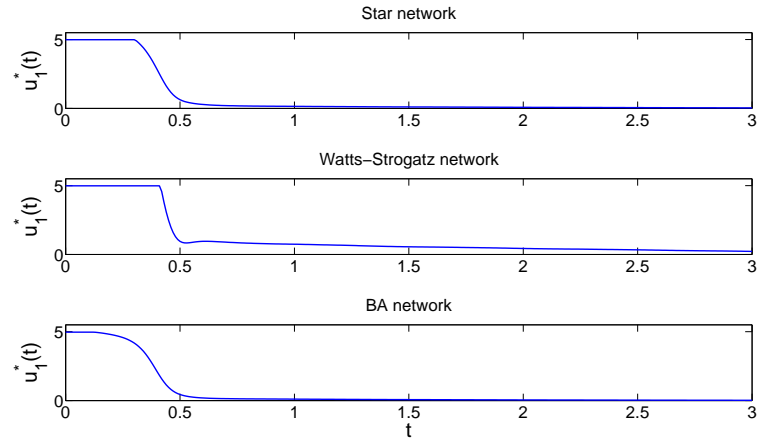

FIG. 4: The dynamical change processes of the optimal controls $u_{1}^{*}(t)$ on different complex networks with the parameters (i), where $u_{i}^{*}(t), i=2,3, \ldots, 50$ have the similar dynamical change processes which are omitted for clarity.

with transversality condition

$$
\begin{aligned}
\lambda_{i}\left(t_{f}\right) & =\frac{\partial}{\partial x_{i}\left(t_{f}\right)}\left(\frac{1}{2} \sum_{i=1}^{n} e_{i}^{* T}\left(t_{f}\right) e_{i}^{*}\left(t_{f}\right)\right) \\
& =e_{i}^{*}\left(t_{f}\right),
\end{aligned}
$$

where $i=1,2, \ldots, n$. Furthermore, under the necessary condition, the optimal control should satisfy

$$
\left.\frac{\partial H}{\partial u_{i}(t)}\right|_{x_{i}(t)=x_{i}^{*}(t), u_{i}(t)=u_{i}^{*}(t)}=\beta_{i} u_{i}^{*}(t)-\lambda_{i}^{T}(t) e_{i}^{*}(t)=0
$$

and $u_{i}^{*}(t) \in U_{a d}$. These restriction conditions give the optimal control

$$
u_{i}^{*}(t)= \begin{cases}0, & \frac{\lambda_{i}^{T}(t) e_{i}^{*}(t)}{\beta_{i}} \leq 0 ; \\ \frac{\lambda_{i}^{T}(t) e_{i}^{*}(t)}{\beta_{i}}, & 0<\frac{\lambda_{i}^{T}(t) e_{i}^{*}(t)}{\beta_{i}}<b ; \\ b, & \frac{\lambda_{i}^{T}(t) e_{i}^{*}(t)}{\beta_{i}} \geq b,\end{cases}
$$

whose compact expression is given by

$$
u_{i}^{*}(t)=\min \left\{\max \left(0, \frac{\lambda_{i}^{T}(t) e_{i}^{*}(t)}{\beta_{i}}\right), b\right\} .
$$

Therefore, the optimal system of the complex dynamical network under the optimal control is determined by equations (1), (6) with initial condition (2), transversality condition (7) and optimal control (10). In general, it is impossible to obtain an analytic state solution and optimal control, however, a solution can be obtained by numerical methods. In the following simulations, we will apply the forward-backward sweep method [16] to solve the optimal system, where the underlying ordinary differential equations (1) and (6) are solved by a fourth order Runge-Kutta method. The control strengths are updated by convex combination between the current strengths and the new control strengths provided by (10).

Without loss of generality, the heterogeneous structure is characterized by three cases: Star network, WattsStrogatz network and BA network. The network size
Watts-Strogatz network

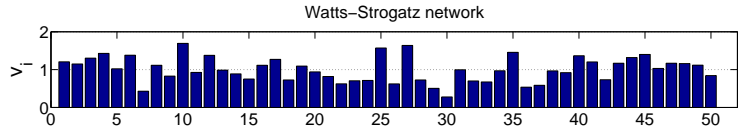

BA network

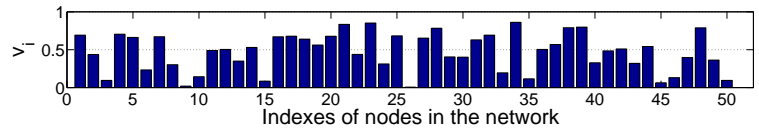

FIG. 5: The average control strengths $v_{i}$ of the optimal control for each node in the network with the parameters (i).

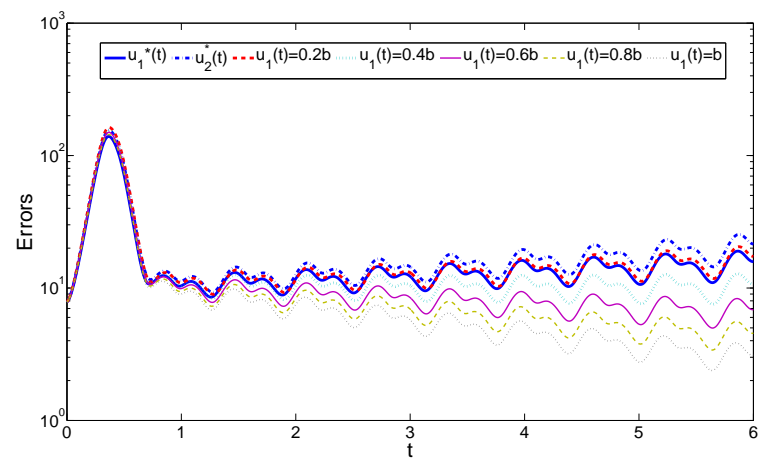

FIG. 6: (Color online) The synchronization errors $e(t)$ on the Star network with the optimal pinning controls and different constant pinning controls on the first node.

is $n$. In the Star network, the first node has degree $n-1$, while the other nodes have the same degree 1 . The WattsStrogatz network [17] is generated with probability 0.1 for rewiring links, where each node is symmetrically connected with its 6 nearest neighbors in its initial nearest neighbor network. The BA network [18] is produced with four initial nodes, which are fully connected, and then adding a new node with three new edges at each time step.

The local dynamics of each node is described by the chaotic Lorenz oscillator

$$
\left\{\begin{array}{l}
\dot{z}_{1}=a_{1}\left(z_{2}-z_{1}\right) \\
\dot{z}_{2}=a_{2} z_{1}-z_{2}-z_{1} z_{3}, \\
\dot{z}_{3}=z_{1} z_{2}-a_{3} z_{3},
\end{array}\right.
$$

where $a_{1}=10, a_{2}=28, a_{3}=8 / 3$. So, we have $m=3$ and

$$
\frac{\partial f(z)}{\partial z}=\left(\begin{array}{ccc}
-a_{1} & a_{1} & 0 \\
a_{2}-z_{3} & -1 & -z_{1} \\
z_{2} & z_{1} & -a_{3}
\end{array}\right) .
$$

In order to show that the choice of parameters for numerical simulations doesn't affect the results, we consider two groups of parameter: (i) $n=50, c=0.1$, 
$b=5$ and $\beta_{i}=1$ for $i=1,2, \ldots, n$; (ii) $n=60$, $c=0.2, b=6, \beta_{i}=0.5$ for $i=1,2, \ldots, n / 2, \beta_{i}=1$ for $i=n / 2+1, \ldots, n$. The initial value $\theta_{i}$ are chosen from $[0,1]^{3}$ at random for $i=1,2, \ldots, n+1$. To show the effectiveness of the proposed optimal control strategy, different constant controls and optimal control are compared via their corresponding synchronization errors and the values of their objective function. Figures 12 show that, though the synchronization error with the optimal control is larger than some constant control algorithms, it is limited to a relatively low level (the error can become smaller by setting larger upper bound $b$ ). That is to say, practical synchronization is realized with the proposed optimal control strategy on these networks. What is more important, from Fig. 3, the optimal control strategy makes the value of objective function $J$ minimal, while the other constant controls all result in larger $J$. In Fig. 4, we can see the dynamical change process of optimal control on different networks. To further measure the global control strengths, we define the average control strengths of the optimal control for each node as

$$
v_{i}=\frac{1}{t_{f}} \int_{0}^{t_{f}} u_{i}^{*}(t) d t, \quad i=1,2, \ldots, n .
$$

Obviously, $0 \leq v_{i} \leq b$, as $0 \leq u_{i}^{*}(t) \leq b$. Figure 5 shows that the average control strengths of the optimal control remain relatively low for each network. Hence, we can conclude that the optimal control indeed realizes a balance between the synchronization error and control strength.

Though the above proposed optimal control is effective in achieving practical synchronization, it may be infeasible in real applications as it needs to control all nodes in the network. In order to further improve the performance of optimal control, we will propose the optimal pinning control strategy by which only a fraction of nodes in the network are controlled. As we known, for synchronization on heterogeneous networks, the targeted pinning scheme, which aims to pin the nodes with largest degree, is more effective than random pinning applied to the same number of nodes $[4-6,19]$. Hence, we expect to improve the performance of optimal control by targeting control on the nodes with large degree.

For simplicity, we take the Star network with size $n=10$ for example to show the implement of optimal pinning control strategy. In fact, Star network is usually investigated for synchronization as it can be considered as a motif of the famous Scale-Free networks [20]. In this case, as the first node has the maximal degree, we will only control this node. To this end, in network $(1)$, let $u_{i}(t)=0, i=2,3, \ldots, n$, then $u_{1}^{*}(t)=\min \left\{\max \left(0, \frac{\lambda_{1}^{T}(t) e_{1}^{*}(t)}{\beta_{1}}\right), b\right\}$ from (10), and $u_{i}^{*}(t)=0, i=2,3, \ldots, n$ in equations (6). The other parameters are set as $c=2, b=5, t_{f}=5$ and $\beta_{1}=1$. The initial value $\theta_{i}$ are chosen from $[0,1]^{3}$ at random for $i=1,2, \ldots, n+1$. From Fig. 6 , we find that the synchronization error under the optimal pinning control $u_{1}^{*}(t)$ is

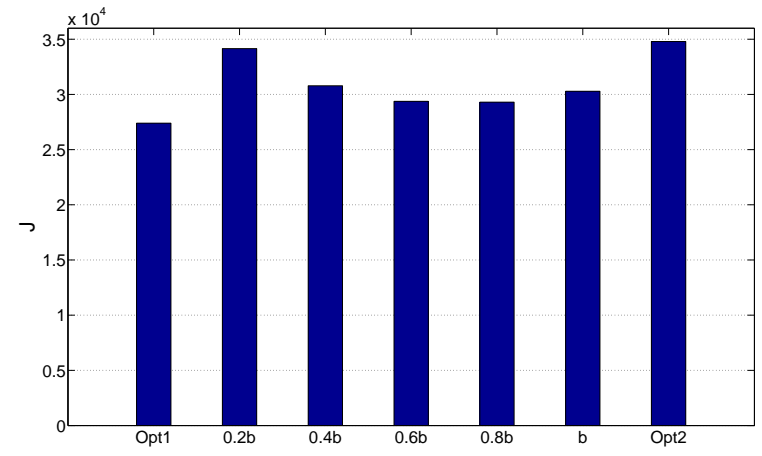

FIG. 7: The values of objective function (3) on the Star network with the optimal pinning control, and different constant pinning controls on the first node with control strengths from $0.2 b$ to $b$. 'Opt1', 'Opt2' denote $u_{1}^{*}$ and $u_{2}^{*}$, respectively.

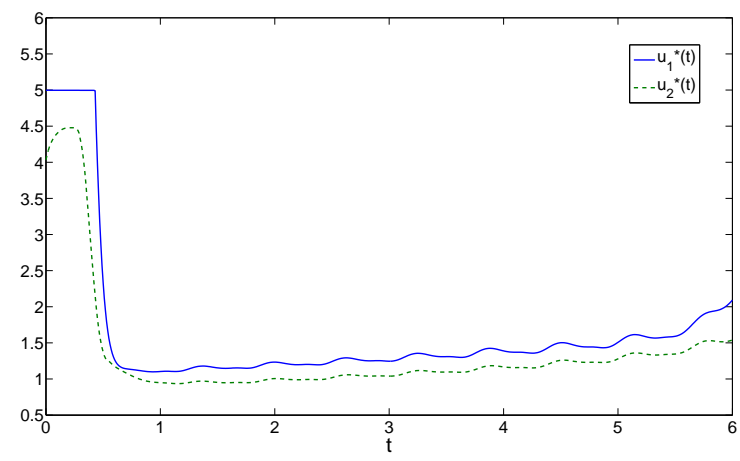

FIG. 8: (Color online) The dynamical change processes of the optimal controls on the Star network with size $n=10$. The average control strengths $v_{1}=1.6494, v_{2}=1.3547$.

limited to a relatively low level, compared to the other constant pinning controls on the same node. Moreover, from Fig. 7, the value of the objective function is minimal when we apply the optimal pinning control. Fig. 8 shows the dynamical change process of the optimal control $u_{1}^{*}(t)$ with small average control strength $v_{1}=1.6494$. For further comparison, we find that the synchronization error and objective function are both increased if only the second node is controlled with optimal control $u_{2}^{*}(t)$ (see Fig. 6-7), where the other parameters are the same as the optimal pinning control. Hence, in real applications of practical synchronization, the optimal pinning control strategy is a highly effective method, as the number of controlled nodes and the objective function are both small. As we know, there is a close relationship between network synchronization and network topological structure. The developed optimal pinning control strategy provides a feasible method to optimize the synchronization control on complex dynamical networks.

This research was supported jointly by NSFC grants (Nos. 61004101, 11331009) and Guangxi Natural Science Foundation Program (Nos.2011GXNSFB018059, 
2014GXNSFBA118007). MS is supported by an ARC

Future Fellowship (No. FT110100896). This work was supported by Guangxi Colleges and Universities Key Laboratory of Data Analysis and Computation, Guilin
University of Electronic Technology. This work was also supported by Program for Innovative Research Team of Guilin University of Electronic Technology.
[1] M. E. J. Newman, SIAM Rev. 45, 167 (2003).

[2] X. F. Wang and G. R. Chen, IEEE Circ. Syst. Mag. 3, 6 (2003).

[3] A. Arenas, A. Diaz-Guilera, J. Kurths, Y. Moreno, and C. S. Zhou, Phys. Rep. 469, 93 (2008).

[4] F. Sorrentino, M. di Bernardo, F. Garofalo, and G. R. Chen, Phys. Rev. E 75, 046103 (2007).

[5] X. F. Wang and G. R. Chen, Physica A 310, 521 (2002).

[6] T. P. Chen, X. W. Liu, and W. L. Lu, IEEE Trans. Circuits Syst. -I 54, 1317 (2007).

[7] B. Liu, X. Z. Liu, G. R. Chen, and H. Y. Wang, IEEE Trans. Circuits Syst. -I 52, 1431 (2005).

[8] J. Zhou, J. A. Lu, and J. H. Lü, IEEE Trans. Automat. Control 51, 652 (2006).

[9] R. Femat and G. Solis-Perales, Phys. Lett. A 262, 50 (1999).

[10] F. M. Moukam Kakmeni, S. Bowong, D. V. Senthikumar, and J. Kurths, Chaos 20, 043121 (2010).

[11] A. El-Gohary, Chaos Soliton. Fract. 27, 345 (2006).
[12] M. Rafikov and J. M. Balthazar, Commun. Nonlinear Sci. Numer. Simulat. 13, 1246 (2008).

[13] F. Motallebzadeh, M. R. J. Motlagh, and Z. R. Cherati, Commun. Nonlinear Sci. Numer. Simulat. 17, 3643 (2012).

[14] Z. Li and G. R. Chen, Phys. Lett. A 324, 166 (2004).

[15] W. H. Fleming and R.W. Rishel, Deterministic and Stochastic Optimal Control (Springer-Verlag, New York, 1975).

[16] B. Buonomo, D. Lacitignola, and C. Vargas-De-León, Math. Comput. Simulat. 100, 88 (2014).

[17] D. J. Watts and S. H. Strogatz, Nature 393, 440 (1998).

[18] A. L. Barabási and R. Albert, Science 286, 509 (1999).

[19] X. Li, X. F. Wang, and G. R. Chen, IEEE Trans. Circuits Syst. -I 51, 2074 (2004).

[20] Y. Zou, T. Pereira, M. Small, Z. Liu, and J. Kurths, Phys. Rev. Lett. 112, 114102 (2014). 